\title{
Combined Minimal Invasive Transdiaphragmatic Resections of Peripheral Colorectal Lung Metastases in Patients Undergoing Laparoscopic Liver Resections
}

\author{
Philip Lerut $^{1}$, Frederiek Nuytens ${ }^{2}$, and Mathieu D'Hondt ${ }^{2}$ \\ ${ }^{1}$ Department of Thoracic and Vascular Surgery, AZ Groeninge, Kortrijk, Belgium; ${ }^{2}$ Department of Digestive and \\ Hepatobiliary/Pancreatic Surgery, AZ Groeninge, Kortrijk, Belgium
}

\begin{abstract}
Background. The management of patients with simultaneously diagnosed colorectal liver and lung metastases (SLLM) remains controversial. A recent study based on an analysis of the LiverMetSurvey demonstrated that patients with SLLM suitable for resection of all metastases have a survival similar to that of patients who undergo removal of isolated liver metastases. ${ }^{1}$ Simultaneous transdiaphragmatic resection of peripheral lung lesions and liver resection by laparotomy has been described previously. ${ }^{2}$ To the authors' knowledge, no previous reports on a similar minimally invasive approach have been published. In April 2015, the authors started performing combined minimally invasive transdiaphragmatic resections of peripheral colorectal lung metastases in patients undergoing laparoscopic liver resections. This video aims to demonstrate the authors' first experience with this minimally invasive approach.

Methods. This report describes a combined minimally invasive transdiaphragmatic resection of peripheral colorectal lung metastasis in a patient undergoing a laparoscopic liver resection. General anesthesia was induced with placement of a double-lumen endotracheal tube to achieve single-lung ventilation. Once laparoscopic liver resection was completed, the left lung containing the metastatic lesion was deflated. The left hemidiaphragm was
\end{abstract}

Electronic supplementary material The online version of this article (doi:10.1245/s10434-016-5304-2) contains supplementary material, which is available to authorized users.

\section{(C) Society of Surgical Oncology 2016}

First Received: 10 May 2016;

Published Online: 14 June 2016

M. D'Hondt

e-mail: mathieudhondt2000@yahoo.com carefully divided using a 10-cm incision around the central tendon to avoid damage to the phrenic nerve. The lung metastasis was localized using intraoperative ultrasound, and the lesion was resected using endoscopic $60-\mathrm{mm}$ vascular staplers. A thoracic tube was placed, and the diaphragm was closed with a running nonabsorbable suture.

Results. The operative time was $180 \mathrm{~min}$, and the blood loss was $100 \mathrm{ml}$. The postoperative course was uneventful. The patient was discharged on postoperative day 4 . Pathology confirmed two colorectal metastases. Tumorfree margins of $5 \mathrm{~mm}$ for the liver and $7 \mathrm{~mm}$ for the lung were achieved.

Conclusions. Simultaneous transdiaphragmatic resection of peripheral lung lesions is feasible for patients undergoing laparoscopic liver resection. The low invasiveness of the described technique could facilitate an aggressive operative approach to SLLM.

ETHICAL APPROVAL All procedures performed were in accordance with the ethical standards of the institutionalresearch committee.

\section{REFERENCES}

1. Andres A, Mentha G, Adam R, Gerstel E, Skipenko OG, Barroso E, et al. (2015) Surgical management of patients with colorectal cancer and simultaneous liver and lung metastases. Br J Surg. 102:691-9.

2. Mise Y, Mehran RJ, Aloia TA, Vauthey JN (2014) Simultaneous lung resection via a transdiaphragmatic approach in patients undergoing liver resection for synchronous liver and lung metastases. Surgery. 156:1197-203. 